\title{
Using Train Interconnection for Intra-train Communication via CAN
}

\section{Tamás Bécsi, Szilárd Aradi}

Budapest University of Technology and Economics, Department of Control for Transportation and Vehicle Systems, Stoczek u. 2, H-1111 Budapest, Hungary, email: becsi.tamas@mail.bme.hu, aradi.szilard@mail.bme.hu

\section{Péter Gáspár}

Computer and Automation Research Institute, Hungarian Academy of Sciences, Kende u. 13-17, H-1111 Budapest, Hungary, e-mail: gaspar.peter@sztaki.mta.hu

\footnotetext{
Abstract: This paper presents the possibilities for using currently installed interconnection cables for extended intra-train communication purposes and proposes a CAN-based communication solution for the problem. The implementation of such a communication technique raises feasibility issues due to the non-standard physical media and the non-fixed network topologies, such as, the determination of an achievable bandwidth, the dynamic termination of the network and the enumeration of units. As a basis of on-board passenger information and telemetry solutions for the operator, this solution could be a cost effective way to improve the quality of service of rail transportation. This paper describes the specialties of the vehicular environment and the proposed network model; it then presents a network lookup algorithm for automated enrolling and ordering the needed train units. Laboratory and field measurements are presented to validate the feasibility of this solution.
}

Keywords: rail transport; vehicle; on-board communication; CAN Network; UIC558

\section{Introduction}

Improving quality of service and reducing maintenance costs, in the area of rail services, are essential to preserve a competitive environment for this transportation mode over the various alternatives. As a consequence of these needs, on-line communication, telemetry and fleet management have gained a significant role in today's modern rail transport. Such systems utilize high-level integration of communication, data management and control systems. Another possibility could include the involvement of intra-train communications within the 
fleet management systems. However, installing new physical communication media in all of the existing units can be very expensive. These facts lead to the idea of using the existing network for the extended communication needs. Multiple train communication networks exist for general train control purposes, such as remote traction control to handle push-pull train operations, door and light control or the audio channels. However, a demand for extended services of intratrain communications has risen. These extended systems can serve several purposes:

- Such systems can provide train specific data to the passenger information system improving passenger satisfaction by providing real-time information about the state of the journey, i.e. current delay, estimated arrival, connections etc. This information could be displayed in passenger cars via various kinds of displays, which obtain data from the on-board unit of the driver's stand.

- Another benefit is the collection of train information for the operator. The telemetry or the automatic enrolment of train units can be centralized at the train level with a closed communication solution, where the only connection to a central data center of the operator is managed by the onboard unit (OBU) of the driver's station.

- The system can improve the safety of railroad operations by the remote monitoring of various mechanical elements of a railcar. [3]

Although a standardized extension of the Train Communication Network (TCN) [7] [8] [9] exists in newer passenger and traction units, which provides additional channels and protocols for intra-train communication, the penetration is low and the need of addressing this problem in current units that are intended to be in service for a long term, is growing at the operator level. There have been various attempts to implement such a communication system on existing interconnections [5] [6].

Naturally, one must examine the pin allocation of the connectors for the current system and recognize wire pairs that do not carry safety-critical information, in order to maintain the safety level of train operation.

Our previous work deals with the theoretical aspects of this communication, via a non-standard physical layer, the non-fixed topology of the network, network length and speed [2].

This paper proposes a solution that uses CAN communications with the interconnection media. Section 2 briefly describes the specialties of the vehicular environment and the proposed network model. Section 3 presents a network lookup algorithm for automated enrolment and ordering of the train units. Section 4 , laboratory and field measurements are presented that validate the feasibility of our solution. 


\section{Digital Data Transfer via Existing Interconnection}

As a consequence, of the existing conditions of the current installation interconnections, the network topology of the train can only form two kinds of basic topologies: the chain and the bus topology as shown in Figure 2. Of these two, bus topology has been chosen in our research, since the implementation of chain topology frequently breaks the continuity of existing interconnections, which is undesirable. In our scope, the application of UIC 558 type 18 pin interconnection [1] is examined.
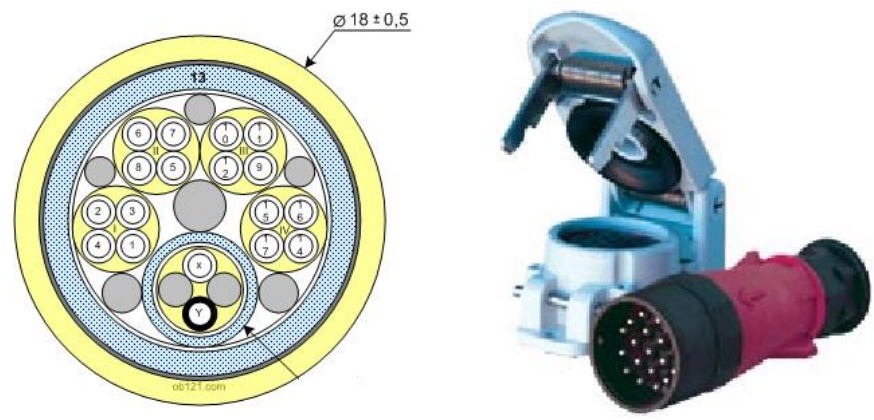

Figure 1

UIC 558 Series Cable and Connector (source: www.hertfordcontrols.co.uk, www.ob121.com)

The function allocation of the UIC 558 wire is shown in Table 1. Theoretically, one could implement digital signal transfer on any wire within interconnection, wires 9-18 transfer safety critical functions and therefore, the utilization of only the audio-related wires is desired.
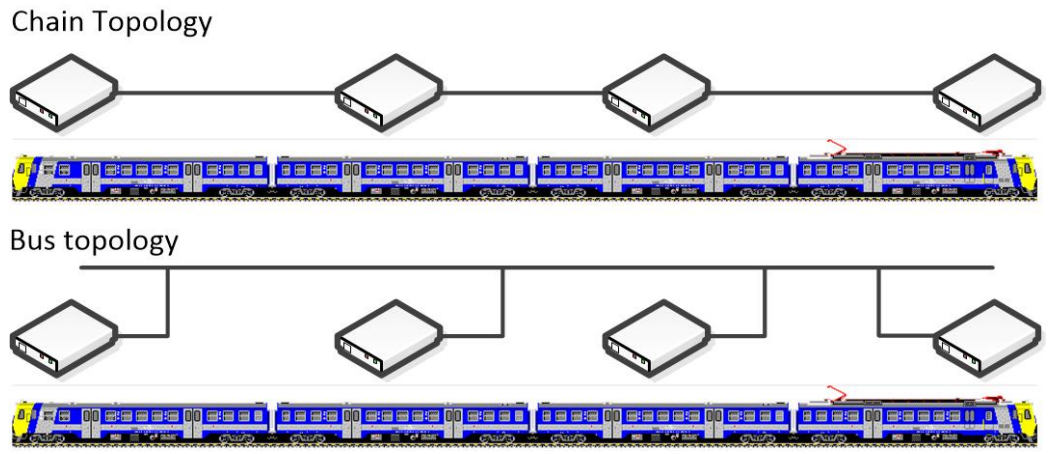

Figure 2

The chain and bus topology 
The network topology of the train is not fixed, since the individual units can form different configurations of, length and in addition, the traction or control units can take any position within the train. These features of a modular network pose several problems:

- The switchable galvanic separation of communication units from the media

- The priority of the original function

- The adequate or even adaptive termination of wire pairs

- The determination of an achievable network speed

- The enumeration enrolment and ordering of units currently on the network

Since our previous work deals with theoretical electronics and data transferrelated problems, the network build, enrolment issues and validation measurements are presented in the following sections.

Table 1

Function allocation of the UIC 558 cables

\begin{tabular}{|l|l|l|}
\hline \multicolumn{2}{|l|}{ Wire Pair } & Function \\
\hline 1 & 2 & Audio towards train loudspeaker \\
\hline 3 & 4 & Voice communication towards: \\
$3(-)$ & $4(+)$ & - the driver \\
$3(+)$ & $4(-)$ & - the switchboard \\
\hline $5(+)$ & $6(-)$ & Switch on loudspeaker amplifiers \\
\hline $7(+)$ & $8(-)$ & Priority of announcement command \\
$7\left(X^{*}\right)$ & $7\left(Y^{*}\right)$ & TCN* Communication \\
\hline $9(+)$ & $12(-)$ & Remote command of door closing \\
\hline $10(+)$ & $12(-)$ & Remote command: light on \\
\hline $11(+)$ & $12(-)$ & Remote command: light off \\
\hline $14(+)$ & $12(-)$ & Unlock right doors command \\
\hline $15(+)$ & $12(-)$ & Unlock left doors command \\
\hline $16(+)$ & $12(-)$ & Remote control of door locked condition \\
\hline $17(\mathrm{X})$ & $18(\mathrm{Y})$ & TCN Communication \\
\hline $\mathrm{S}$ & & Shield of wires 17-18 \\
\hline 13 & & Common shield for all wires \\
\hline
\end{tabular}

\section{Network Look-Up Algorithm}

As mentioned before, knowing the order of the units in the train, is essential since the correct positioning of the termination, in the CAN network, highly increases the optimum bandwidth. On the other hand, the determination of the order of the 
devices in the network also serves an operational purpose, by enabling an adequate automatic enumeration, of the train's units. For this task, a suitable network lookup algorithm is needed.

As a consequence of the given conditions, the train has a bus network topology for the CAN communications, as shown in Figure 3. It can be seen that all on-board units, of the train's unit, connect to a common two-wire bus provided by the interconnection cables. The problem is that the topology is not constant, since the composition of the train can be freely manipulated by the operator.

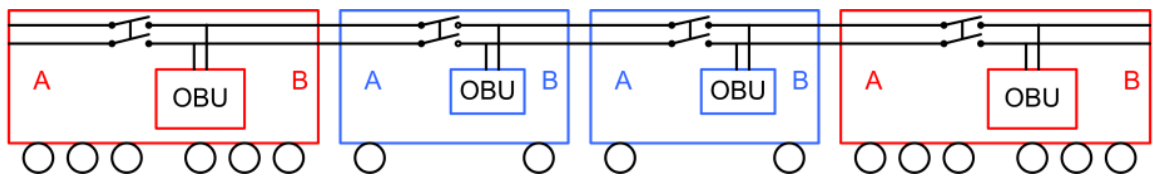

Figure 3

Network topology scheme

To enable the determination of the order of the units, the interconnection cable has a circuit breaker for all OBUs. Since the circuit breaker also prevents the original function of the wire pair from operating, it must be designed to a high safety standard and have the ability to close, whenever the original functionality is needed. These switches are normally in closed state and are only openi during the execution of the network lookup algorithm.

The basic concept behind the algorithm is the following:

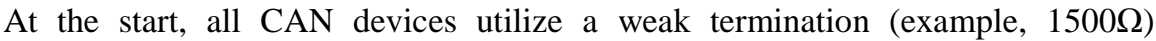
enabling approximately $1 \mathrm{kbit} / \mathrm{s}$ bandwidth on the network, even with a relatively long bus. With this bus speed, the number, position and orientation of each unit can be determined. With this knowledge the network can be adequately terminated at the ends of the train.

Since the media access of the CAN network is based on arbitration, there is no master role on the network. Thus to control the network lookup algorithm a dedicated OBU must be chosen for the task. This way, communications on the network, has the following stages (see Figure 4):

- Normal communication

- Network Lookup

- Disconnected (Media used by original function)

Since the wire pair chosen for communication is rarely used, it is considered to be normal state, when all switches are closed and all OBUs are communicating on the network. This state can be interrupted in two ways: one is when the original function needs the media line and the other is when a new OBU appears on the network. 


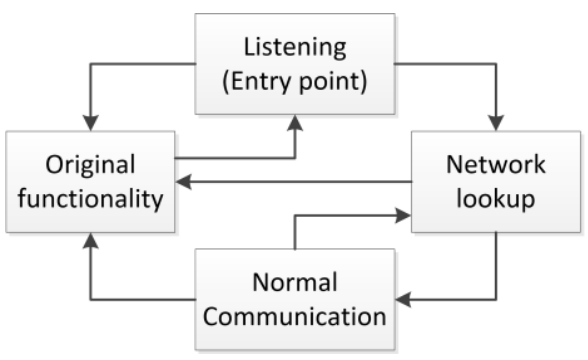

Figure 4

Network state diagram

When the original function needs the media, the OBUs leave their switches closed, but disconnect their transceivers from the network.

When a new unit ID appears on the network the network lookup algorithm starts.

First, all OBUs send their unique identifiers on the network. The one with the lowest value is chosen as the master for communication.

During the network lookup phase, all units, including the master, continuously send their IDs on the network, and their order is determined by their visibility, depending on the state of the circuit breakers.

The master systematically sends a message to each OBU (including to itself) to temporarily open its circuit breaker. This message is available for any unit on the network, so all OBUs know which unit opens the media separating the network into two halves. For this short time, all OBUs receive only the IDs of those units which are on the same side of the network. This way, all OBUs will have a set of visible IDs for each opened breaker.

Figure 5 shows a simple example for this systematic enumeration. It shows the algorithm, from the point of view, of the left-side unit with two additional units having different orientations. It can be seen that with any combination of the orientations, of the two units, their visibility is different according to the state of the switches and so both their order and orientation can be determined.

After acquiring a complete set of visible OBUs, for all opened switches, every OBU can determine the order and orientation with two easy steps:

- First it determines the orientation of each OBU, by examining the set generated, when the given unit's circuit breaker was opened. If the unit's ID is contained in this set, it means that its transceiver is closer than its circuit breaker.

- Second the unit excludes all visible unit IDs from their sets, and orders the set with a sorting algorithm, that gives the order of the units. Naturally, with OBUs in the middle of the train, the units must be handled differently on their two respective sides. 

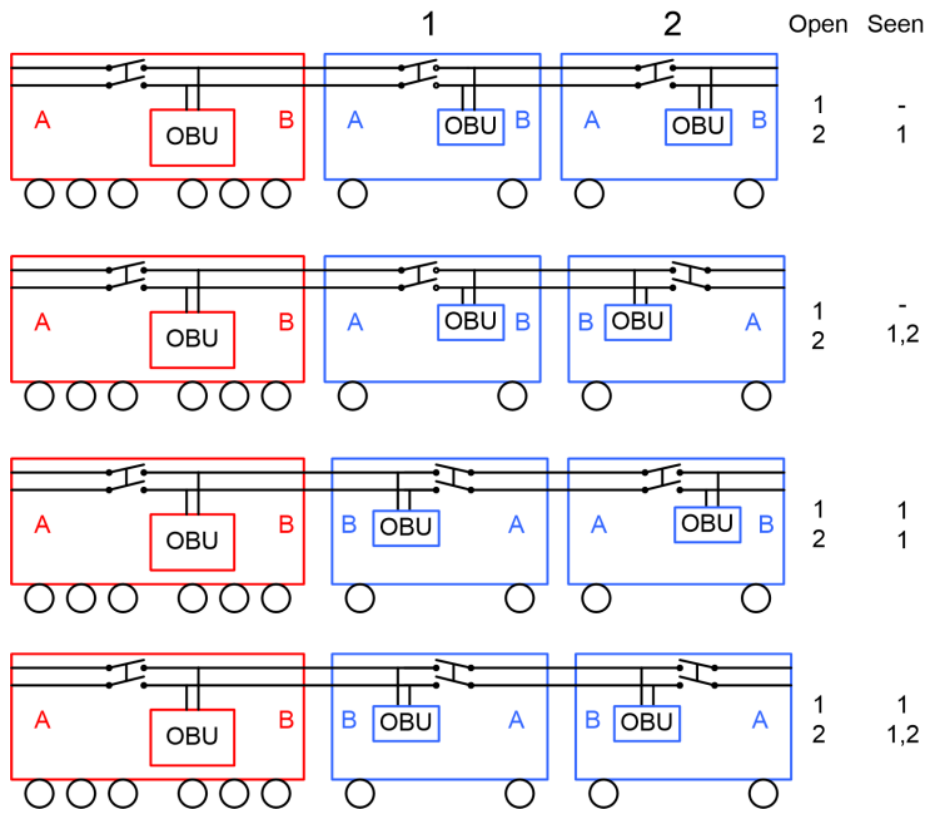

Figure 5

Network lookup example

Table 2 shows an example with a unit train, from the view of the left side end of the network. For the simplicity of the example the OBUs' order and their IDs' are identical and the "Spin" column shows the orientation of the unit. Spin value 0 means that the circuit breaker is on the left of the CAN transceiver and a value of 1 means it is on the right. It is shown that only the IDs of the units with the spin of 1 (3, 4, 8 and 9) appear in their corresponding sets. By excluding these identifiers the cardinality of set, determines the distance from the left end.

Table 2

Example network lookup results of a nine unit train

\begin{tabular}{|c|c|l|}
\hline \multicolumn{1}{|l|}{ Spin } & \multicolumn{1}{l|}{ ID } & Visible \\
\hline 0 & 1 & \\
\hline 0 & 2 & 1 \\
\hline 1 & 3 & $1,2,3^{*}$ \\
\hline 1 & 4 & $1,2,3, \mathbf{4}^{*}$ \\
\hline 0 & 5 & $1,2,3,4$ \\
\hline 0 & 6 & $1,2,3,4,5$ \\
\hline 0 & 7 & $1,2,3,4,5,6$ \\
\hline 1 & 8 & $1,2,3,4,5,6,7, \mathbf{8}^{*}$ \\
\hline 1 & 9 & $1,2,3,4,5,6,7,8, \mathbf{9}^{*}$ \\
\hline \hline & None & $1,2,3,4,5,6,7,8,9$ \\
\hline
\end{tabular}


Let us define the set of all visible identifiers as $A$ and the set of the IDs from the point of view of the unit with the ID $i$ when the circuit breaker of the $j^{\text {th }}$ unit is opened as $\mathrm{A}_{\mathrm{i}}^{\mathrm{j}}$. If we state that the circuit breaker of the $i^{\text {th }}$ unit is on the left of its transceiver then the units on its right side are:

$$
U_{i}^{r i g h t}=A_{i}^{i}
$$

and the units on its left:

$$
\mathrm{U}_{\mathrm{i}}^{\text {left }}=\mathrm{A}, \quad \mathrm{A}_{\mathrm{i}}^{\mathrm{i}}
$$

The determination of the orientation of any unit depends on its position according to unit $i$, see Table 3:

Table 3

Determination of the orientation of each unit

\begin{tabular}{|l|l|l|}
\hline & $\mathrm{j} \in \mathrm{U}_{\mathrm{i}}^{\text {left }}$ & $\mathrm{j} \in \mathrm{U}_{\mathrm{i}}^{\text {right }}$ \\
\hline $\mathrm{j} \in \mathrm{A}_{\mathrm{i}}^{\mathrm{j}}$ & Right (1) & Left (0) \\
\hline $\mathrm{j} \notin \mathrm{A}_{\mathrm{i}}^{\mathrm{j}}$ & Left (0) & Right (1) \\
\hline
\end{tabular}

The order can be determined from the distance of each unit, which can be formally written as:

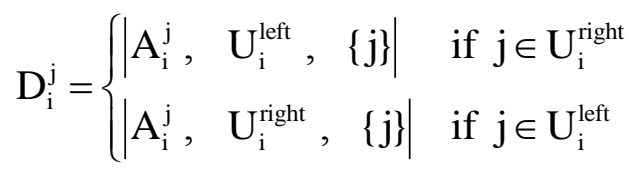

Another issue is the switching between different data transfer rates, on the CAN network. Generally, when one transceiver with a different bandwidth setting connects to a CAN network it automatically detects the messages as erroneous, disrupts the data transfer and begins to send error frames to the network. This undesired phenomenon can be avoided by using the "listening" or the "listen-only" modes of the CAN devices [4]. After start-up, the OBUs can initiate their CAN controller to the listen-only mode and listen alternately in the two possible frequencies and detect the speed of the network. If no communication is taking place, they start with low bandwidth.

Special cases exist when network connection and disconnection events occur:

1 If any disturbance occurs on the network resulting in erroneous network operation, the devices can detect it and fall back to the listening/low bandwidth mode. 
2 When one or more units disconnect, their periodic identifiers stop appearing on the network. In this case, the device with the currently highest ID priority sends a signal to perform the network lookup.

3 The same algorithm occurs when new identifiers appear on the network.

4 When two working networks are connected the termination resistance halves because of their parallel configuration. This is the operational boundary of the CAN network. Depending on the current conditions this can result in either an erroneous or a correctly working network. If the communication on the network remains, this case leads to case 2. When this connection disrupts the communication, it leads to case 1.

5 When the train units are disconnected the termination could be ineligible leading to case 1 .

6 When a device restarts, it stops sending its identifier for a short period of time. If the identifier reappears in a previously defined time there is no need for network lookup.

\section{Experimental Results}

Two tests have been carried out to validate the feasibility of the theory. Since the proposed solution utilizes a non-standard termination of the CAN network, the achievable data transfer rates were tested under laboratory conditions. The physical media of the network is an unshielded twisted pair (UTP Cat5) cable. On one hand, the wire pairs of the interconnection cables are also twisted, although their electrical parameters are different. So, the purpose of the laboratory tests, were the determination of the effect of the non-standard termination.

Along with the standard termination, where $120 \Omega$ terminator resistors were applied at either end of the network, three other terminator resistor configurations were tested:

- $\quad 120 \Omega$ resistor at one end (120/-)

- No termination (-/-)

- $1500 \Omega$ resistors at both ends $(1500 / 1500)$

Figure 6 shows the results of the test. This measurement has clearly shown that the CAN network operates with a non-standard termination, although on a lower bandwidth. 


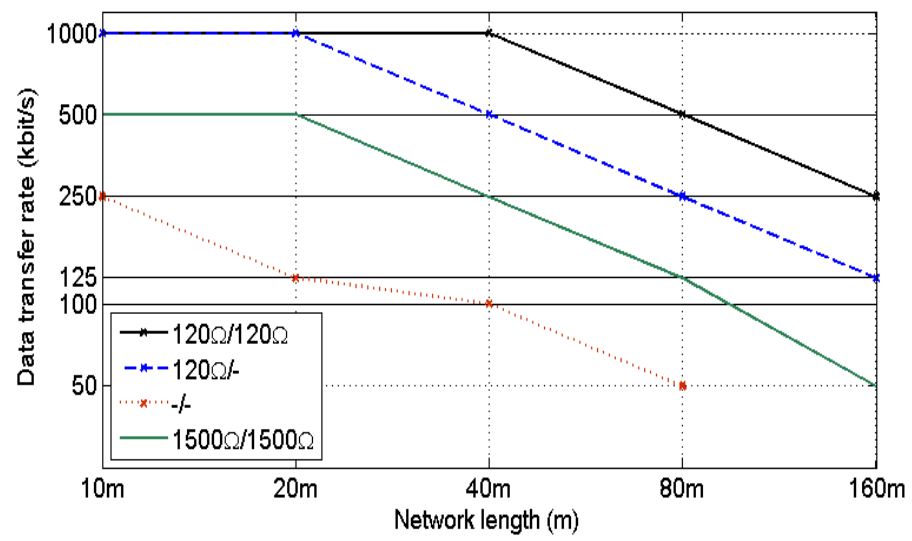

Figure 6

Achievable CAN bandwidth with different termination values

To examine the behavior of the concept under real conditions, a field test was organized with the support of the Hungarian State Railways. The train consisted of 10 - Bhv type passenger cabs and 1 - Bdt type control car, with all units having the same length of $23.740 \mathrm{~m}$. The control car was placed in the middle of the train and the units were connected by an 18-pole UIC558 type interconnection cables. First, as a proof of concept, basic measurements were conducted on a stopped train. After that, the validation of operability, the network reliability tests were performed under a real-world environment test, while the train was moving.

Ten units were involved in the test, all having switchable $1500 \Omega$ resistors and two $120 \Omega$ switchable resistors installed in the 1st and 10th unit. The wire pair used for the communication was:

- 5. Switch on loudspeaker amplifiers (+) for CANH

- 7. Priority of announcement command (+) for CANL

Two CAN analyzers were installed in the $1^{\text {st }}$ and $10^{\text {th }}$ car to generate and measure network load. The distance between the two can devices was approximately 230 meters. CAN frames with extended IDs and 8 byte length data field were sent on the network, with different data values, for example $(0 \times 55)$ for alternating bits and (0x00) for long unchanged state, where bit-stuffing occurs. The average network load was at least $60 \%$ for each measurement, with the maximum value of $80 \%$.

Table 4 summarizes the measurement results. The same measurements were made between the $1^{\text {st }}$ and $2^{\text {nd }}$ and the $1^{\text {st }}$ and $5^{\text {th }}$ units. It can be stated, as a general conclusion, that the CAN technology is sufficiently robust for communication purposes, via train interconnection, even with high length and non CAN-standard cabling and termination. 
Table 4

Actual achievable CAN data transfer rates using UIC 558 interconnection

\begin{tabular}{|l|c|c|c|c|}
\hline \multicolumn{1}{|c|}{ CAN Termination } & $\begin{array}{c}\text { Bus } \\
\text { speed } \\
{[\mathbf{k b i t} / \mathbf{s}]}\end{array}$ & $\begin{array}{c}\text { Average } \\
\text { Bus } \\
\text { Load }\end{array}$ & $\begin{array}{c}\text { Ratio of } \\
\text { erroneous } \\
\text { frames }\end{array}$ & $\begin{array}{c}\text { Network } \\
\text { Operable }\end{array}$ \\
\hline $1500 \Omega$ in every unit & 100 & 69 & 0 & Yes \\
\hline $1500 \Omega$ in every unit & 125 & - & - & No \\
\hline \hline $120 \Omega$ at two ends & 125 & 68 & 0 & Yes \\
\hline $120 \Omega$ at two ends & 250 & - & - & No \\
\hline \hline $120 \Omega / 1500 \Omega$ at two ends & 125 & 68 & 0.019 & Yes \\
\hline $120 \Omega / 1500 \Omega$ at two ends & 250 & - & - & No \\
\hline \hline $1500 \Omega$ at two ends & 20 & 60 & 0 & Yes \\
\hline $1500 \Omega$ at two ends & 50 & - & - & No \\
\hline
\end{tabular}

Based on the measurements it can be assumed that CAN communications with a $20-50 \mathrm{kbit} / \mathrm{s}$ bandwidth, can be safely applied on the existing train interconnection solutions of railways.

\section{Conclusions}

This paper has proposed the utilization of a UIC558 type interconnection for extended communications purposes. The problems arising from this method are diverse, due to the safety constraints of the existing communication media. The adequate design of the non-standard physical layer, for the CAN communications, the switching between network states, the handling of the network control and the enrolment algorithm all need an optimal approach. The benefits that can be obtained from these solutions are significant. In addition to the current railwayspecific applications, the measurements of the non-standard CAN network can be applied in any other field, with ordered electrical constraints, and the results can be utilized in other systems.

The laboratory and field measurements have proven the feasibility of our solution, from the view, of an achievable stable data transfer. It can be stated, that this solution is feasible with long train units, without using any repeaters or signal amplifiers. The network lookup algorithm presented in this paper is sufficiently fast, for the needs of the application, since the slower component, when the circuit breakers are operated, has a linear $\mathrm{O}(\mathrm{n})$, while the sorting algorithm proceeding, has a quadratic $\mathrm{O}\left(\mathrm{n}^{2}\right)$ (worst-case) complexity.

\section{Acknowledgement}

The research has been conducted as part of the projects TÁMOP-4.2.2.A11/1/KONV-2012-0012: 'Basic research for the development of hybrid and electric vehicles' and TÁMOP-4.2.2.C-11/1/KONV-2012-0012: 'Smarter Transport - IT for co-operative transport system'. The projects are supported by the Hungarian Government and co-financed by the European Social Fund. 


\section{References}

[1] MAV-MI UIC 558:1999, Hungarian State Railways Technical Guideline, 1999

[2] Aradi, Sz., Bécsi, T., and Gáspár, P. Development of Vehicle On-Board Communication System for Harsh Environment. Acta Technica Jaurinensis, 6(3):53-63

[3] Edwards, M. C., Donelson, J., Zavis, W. M., Prabhakaran, A., Brabb, D. C., Jackson, A. S., Improving Freight Rail Safety with On-Board Monitoring and Control Systems, Rail Conference, 2005. Proceedings of the 2005 ASME/IEEE Joint, Vol., No., pp. 117-122, 16-18 March 2005

[4] Koppe, U. Automatic Baudrate Detection in Canopen Networks. Proceedings of the $9^{\text {th }}$ International CAN Conference, 2003

[5] Rodriguez-Morcillo, C., Alexandres, S. and Munoz, J. D. Broadband System to Increase Bitrate in Train Communication Networks. Computer Standards \& Interfaces, 31(2):261-271, 2009

[6] Russo, D., Gatti, A., Ghelardini, A., Mancini, G., Verduci, A., Amato, D., Battani, R.: Power Line Communication: a New Approach for Train Passenger Information Systems. $8^{\text {th }}$ World Congress on Railway Research Proceedings, 1(1), 1993

[7] Schäfer, C., Hans, G. IEC 61375-1 and UIC 556-International Standards for Train Communication. In Vehicular Technology Conference Proceedings, VTC 2000-Spring Tokyo, 2000 IEEE 51 ${ }^{\text {st }}$, Volume 2, pp. 1581-1585, 2000

[8] Zeltwanger H. Canopen in the Application Field of Rail Vehicles. Railway Pro, 2010

[9] Zeltwanger, H. Canopen on Track. CAN in Automation e. V., 2012 